DOI 10.31489/2019No2/48-54

UDC 532.529

\title{
INVESTIGATION OF TWO PHASE FLOW MOTION WITH SMALL-SIZE GAS BUBBLES
}

\author{
Vasenin I.M., Narimanov R.K., Perchatkina E.V., Shrager L.A. \\ Tomsk State University, Tomsk, Russian Federation, ring_0@mail.ru
}

\begin{abstract}
The motion of a two-phase gas-liquid jet with small-size bubbles in an ozonizer has been calculated. The mathematical model used in the calculations, unlike the model of interpenetrating continuums for a two-phase medium, does not contain small parameters for derivatives. By virtue of the consideration of the medium compressibility and the dependence of the density on the concentration of bubbles, this model automatically takes into account the processes causing free convection in the gravity field in the presence of the heterogeneous concentration of bubbles. Parametric studies based on the proposed model have been conducted. The efficiency of this approach has been shown. The acceleration of the floating of a gas jet due to its involvement in the motion of the carrier medium has been confirmed. The parameters of the process of dissolution of ozone in the contact tank have been determined, the efficiency of the location of dispersants has been evaluated.
\end{abstract}

Keywords: two-phase flow, gas-liquid medium, small-size bubbles, water ozonation.

\section{Introduction}

The movement of gas-liquid mixtures is ubiquitous in nature, and in a variety of technological processes. One of the urgent technological processes of increasing interest is the disinfection of water in water supply systems by its ozonation [1-3]. If we analyze the use of ozone for water treatment, then two main stages can be identified. At the first stage, ozone was used mainly at the final stage of water purification as an effective disinfectant, which gives transparency to the water, deprives of odor, increases the content of dissolved oxygen, etc.

The second stage is connected with the research results of the last ten years, when they began to work out various options for the use of ozone and at the intermediate and initial stages of water purification. With multi-stage ozonation technology with combined cleaning methods, it will be possible to maximize the removal of various types of contaminants. However, it is necessary to optimize the entire technological process, first of all, with respect to the level of ozone and other reagents at each stage.

The equations of water-air mixtures are periodically considered in the scientific literature in various issues. However, it is authors' opinion that the flow of such mixtures in the field of gravity with the combined effect of free and forced convection needs further investigating. The relevance of the development of gas-liquid mixture models is undeniable. Vasenin et al. [4-5] proposed a physical and mathematical model of the motion of a two-phase mixture with small particles.

This work aimed at the calculation of a two-phase flow of water and small-size bubbles in relation to the problems similar to the problem of ozonation of water in contact tanks.

\section{Physical task description}

The movement of bubbles in a body of water is considered as the convective movement of small particles with a density much lower than the density of water. The physical basis of this convection is very simple. By means of the Archimedes buoyant force a lighter mixture containing a larger amount of gas floats in a heavier fluid in the same way as light warm air floats in a cold environment. The mathematical model for small-size bubbles is simpler than the equations of two- 
phase fluid flow with arbitrary size bubbles and allows us to significantly simplify the problem. The following assumptions were used:

- The movement of each bubble can be considered as the movement of a spherical particle. To satisfy this requirement the bubbles, floating up in water under the action of Archimedes buoyant force, should have a size smaller than $2-3 \times 10^{-3} \mathrm{~m}$.

- The temperature of the bubbles moving in water is equal to the ambient temperature. The estimates [6] show that for air bubbles with a diameter less than $2.5 \cdot 10^{-3} \mathrm{~m}$ the relaxation time of the air and water temperature difference does not exceed $0.1 \mathrm{~s}$. Since the time of small-size bubble staying in the water mass of a contact tank is $\sim 20 s$, the temperature difference between bubbles and water can be neglected.

- The third requirement for small-size bubbles is as follows. The transient time of the velocity of bubbles floating up in water should be negligible compared with the characteristic time of their motion in the whole region.

Implying these conditions, the convective flow with small-size bubbles is described by the mathematical model of convective flow with small particles that differ in density from the carrier medium. The mathematical model of convective flow with small particles is described in [4] in application to the case of a "water-solid particle" mixture. However, the presence of dissolving gas bubbles of variable volume in liquid allows us to consider the gas-liquid mixture as a compressible medium. A detailed description of the derivation of these relations and the validity of the assumptions used are presented in [5]. Based on these assumptions, the equations of motion are derived

$$
\begin{aligned}
& \frac{\partial(\bar{u})}{\partial t}+\overline{\boldsymbol{U}} \nabla \bar{u}+\frac{1}{\bar{\rho}_{l}+\bar{\rho}_{s}} \frac{\partial \bar{p}}{\partial \bar{x}}=\frac{1}{R e} \Delta \bar{u}+\frac{1}{F r} \frac{|\bar{g}|}{\bar{g}} \\
& \frac{\partial(\bar{v})}{\partial t}+\overline{\boldsymbol{U}} \nabla \bar{v}+\frac{1}{\bar{\rho}_{l}+\bar{\rho}_{s}} \frac{\partial \bar{p}}{\partial y}=\frac{1}{R e} \Delta \bar{v}+\frac{1}{F r} \frac{|\bar{g}|}{\bar{g}} \\
& \frac{\partial(\bar{w})}{\partial t}+\overline{\boldsymbol{U}} \nabla \bar{w}+\frac{1}{\bar{\rho}_{l}+\bar{\rho}_{s}} \frac{\partial \bar{p}}{\partial \bar{z}}=\frac{1}{R e} \Delta \bar{w}+\frac{1}{F r} \frac{|\bar{g}|}{\bar{g}},
\end{aligned}
$$

where $\mathrm{p}, \mathrm{U}, \mathrm{u}, \mathrm{v}, \mathrm{w}, \rho \mathrm{s}, \rho \mathrm{l}, \mathrm{Re}, \mathrm{Fr}, \mathrm{g}$ - pressure, velocity vector, velocity components, density of air, density of medium, Reynolds number, and Froude number and a gravity vector, respectively.

The equation of state

$$
P\left[\frac{1}{\rho}-\frac{z}{\rho^{*}\left(1+\frac{P}{k}\right)}\right]=\bar{R} T .
$$

According to the estimation of the motion parameters in the ozonizer, the temperature of the mixture is constant and equal to the temperature of inflow water.

$$
T=T_{0}
$$

To close the system of equations, it is necessary to add the equation of conservation of mass of a two-phase mixture

$$
\frac{\partial\left(\bar{\rho}_{l}+\bar{\rho}_{s}\right)}{\partial t}+\nabla\left(\bar{\rho}_{l}+\bar{\rho}_{s}\right)=0,
$$

and the equation of bubbles mass conservation 


$$
\frac{\partial \bar{\rho}_{s}}{\partial t}+\nabla \bar{\rho}_{s}=0
$$

The presented equations for given initial and boundary conditions make it possible to calculate the parameters of a two-phase flow in a contact tank, time and the trajectory of the movement of the bubbles filled with an ozone-air mixture.

\section{Determination of dependence of the ozone dissolution}

Though, we need another formula to calculate the dissolution of ozone in the bubbles. The mass concentration of ozone in bubbles $m_{o 3}$ obeys the conservation law.

$$
\frac{d\left(V \cdot m_{03}\right)}{d t}=-q
$$

where $\frac{d}{d t}$ - time derivative along the bubble trajectory; $V$-its volume; $q$ - ozone flow into water through its surface.

We assume that the flow $q$ is proportional to a bubble surface $\sigma$ and to the difference in the concentrations of ozone in a bubble and in surrounding water

$$
q=\alpha \sigma\left(m_{\text {оз }}-m_{\text {оз } 0}\right),
$$

where $m_{\text {оз } 0}-$ ozone concentration in water.

Substituting (2) in (1) we obtain

$$
\frac{d\left(V \cdot m_{\text {оз }}\right)}{d t}=-\alpha \sigma\left(m_{\text {оз }}-m_{\text {оз } 0}\right) \text {. }
$$

When rising to the surface of the reactor, the volume of the bubble grows, and therefore the value $V$ cannot be taken out of the sign of derivative. To simplify (3), we suppose water is not saturated with ozone, so we can assume $m_{о з 0}=0$. Introducing the mass of ozone in the bubble $M_{\text {оз }}=m_{03} V$, from (3) we derive the equation

$$
\frac{d M_{\mathrm{o3}}}{d t}=-\frac{\propto \sigma}{V} M_{\mathrm{o} 3}
$$

The following equality holds for a spherical bubble $\frac{\sigma}{V}=\frac{3}{2 D}$, where $D$ - bubble diameter. Therefore, equation (4) can be rewritten as

$$
\frac{d M_{\mathrm{o3}}}{d t}=-\frac{3 \propto}{2 D} M_{\mathrm{o} 3}
$$

In the case of constant $D$ and $\alpha$, this equation has a solution

$$
M_{\text {оз }}=C \exp \left(-\frac{t}{T^{*}}\right)
$$

where $T^{*}=2 D / \alpha-$ characteristic time during which the mass of ozone in a bubble changes e times.

Equation (5) was used in the mathematical model described above to calculate the ozone concentration along the trajectories of bubbles. In this case, the variability of bubble diameters D has been considered. The coefficient $\alpha$ was chosen so that for bubbles with an initial diameter of $D_{0}=1.510^{-3} \mathrm{~m}$ in the conditions of the experimental contact tanks of the Eastern water station in 
Moscow an ozone absorption coefficient of $90 \%$ was achieved. Following this approach, the coefficient $\alpha=0.00018 \mathrm{~m} / \mathrm{s}$ was found. For the bubbles with a diameter of $D_{0}=1.510^{-3} \mathrm{~m}$ and $\alpha=0.00018 \mathrm{~m} / \mathrm{s}$, the characteristic time is $\mathrm{T} * \approx 8.3 \mathrm{~s}$. This result is consistent with the time of dissolution of the same air bubble in air-saturated water, which, according to [7], is $7.7 \mathrm{~s}$. A slightly longer dissolution time of ozone may be described by its larger molecular weight, which reduces the diffusion coefficient.

\section{Numerical simulation}

\subsection{Calculation of the movement of a single gas-liquid jet}

Based on the proposed model, the parametric studies were carried out. They include parametric studies of the effect of the initial bubble density and inlet flow on the bubbles rate at which they reach the upper boundary of the cylindrical region. The region is a vertical axisymmetric cylinder; a gas jet is introduced on the cylinder axis. The height and the radius of the cylinder is $10 \mathrm{~cm}$, the width of the jet is equal to $1 / 6$ of the radius. The initial distribution of the bubbles volume concentration in the inlet is determined by a Gaussian probability function so that at the distance of a jet radius from the centerline their concentration is $1 \%$ of the maximum value on the axis. The reliability of calculations is confirmed by the investigation of grid convergence for the bubble sizes varying from $10 \mu \mathrm{m}$ to $100 \mu \mathrm{m}$ at a given initial concentration of the jet. Resizing of a bubble during its floating is neglected.

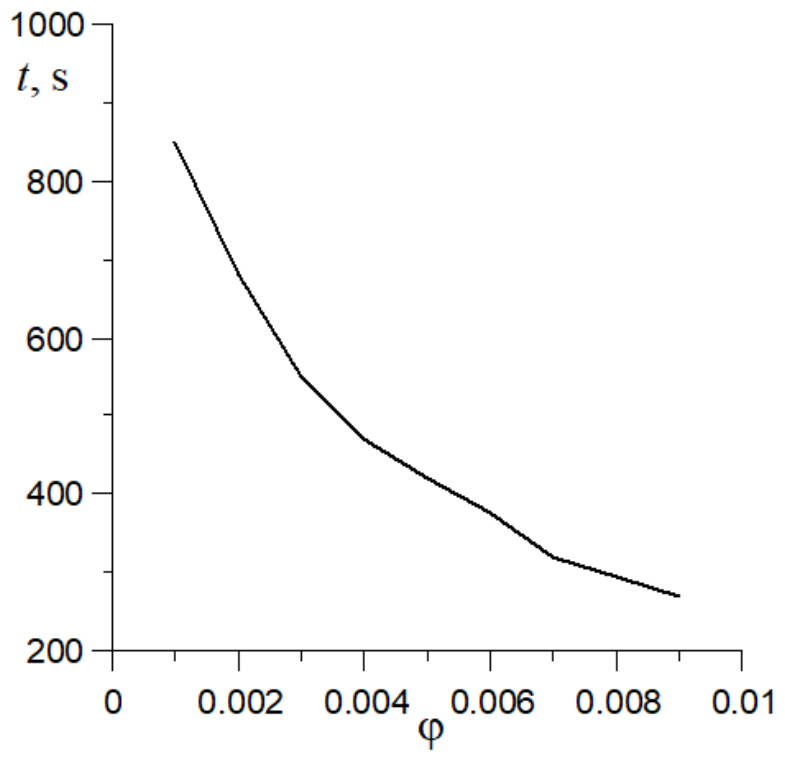

Fig.1. Effect of the introduced relative gas density on the time of reaching the upper boundary

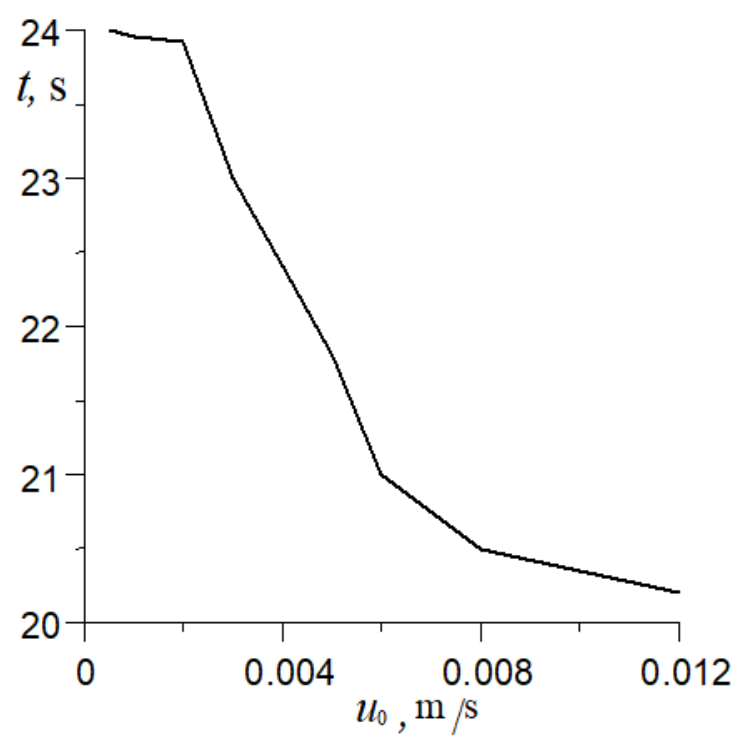

Fig.2. Dependence of the rise time on the perturbation velocity

Figure 1 shows the effect of the relative density of introduced gas $\varphi$ on the time of reaching the upper boundary; here a particle diameter is $10 \mu \mathrm{m}$. When calculating the gas velocity, it is necessary to set a low initial injection velocity, which is parallel to the axis. The value of the velocity $u_{0}$, which does not alter the calculation results, was determined during parametric studies of the dependence of the floating time on the perturbation velocity at the diameter of $100 \mu \mathrm{m}$.

The result is presented in Figure 2. Figure 3 demonstrates the effect of a gas bubble diameter on its floating time at the fixed volume concentration of bubbles in a gas stream. 


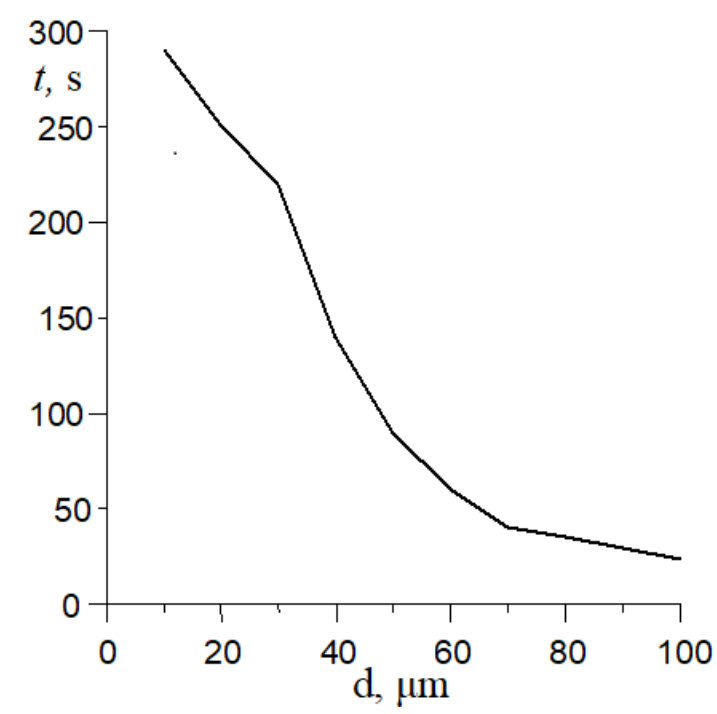

Fig.3. The effect of the diameter of a gas bubble on its ascent time

\subsection{Parametric studies of ozone dissolution in a contact tank}

Preliminary numerical investigations of the process of ozone dissolution in water have been carried out with the following purposes:

1) to validate the proposed mathematical model;

2) To find the ways to optimize and to improve the efficiency of ozonation;

3) To select the diffusion coefficient of ozone for bubbles in a body of water, ensuring the consistency of theory and experimental data.

The calculation of the flow of a two-phase mixture has been carried out for a section of the tank with a horizontal cross-sectional area of $1.6 \mathrm{~m} \times 1.6 \mathrm{~m}$, since there is symmetry in the arrangement of dispersants in the contact tank. At the bottom of the section there are 25 dispersants with a diameter of $0.2 \mathrm{~m}$. Water is delivered or drained between the dispersants. In this section water rate through $1 \mathrm{~m}^{2}$ and the volume of the ozone-air mixture through dispersants are equal to the same parameters in the contact tank.

The axis of the coordinate system is directed from the surface of the tank toward the bottom. On the surface of the tank the atmospheric pressure is set. At the lateral boundaries of the computational domain, symmetry conditions are specified. The calculations showed that the jet stream of the ozone-air mixture will remain throughout the calculations until the establishment of the process. Figure 4 shows the calculation results of a single jet of ozone-air mixture flowing into the counter flow of water from a dispersant, which is $0.6 \mathrm{~m}$ in diameter.

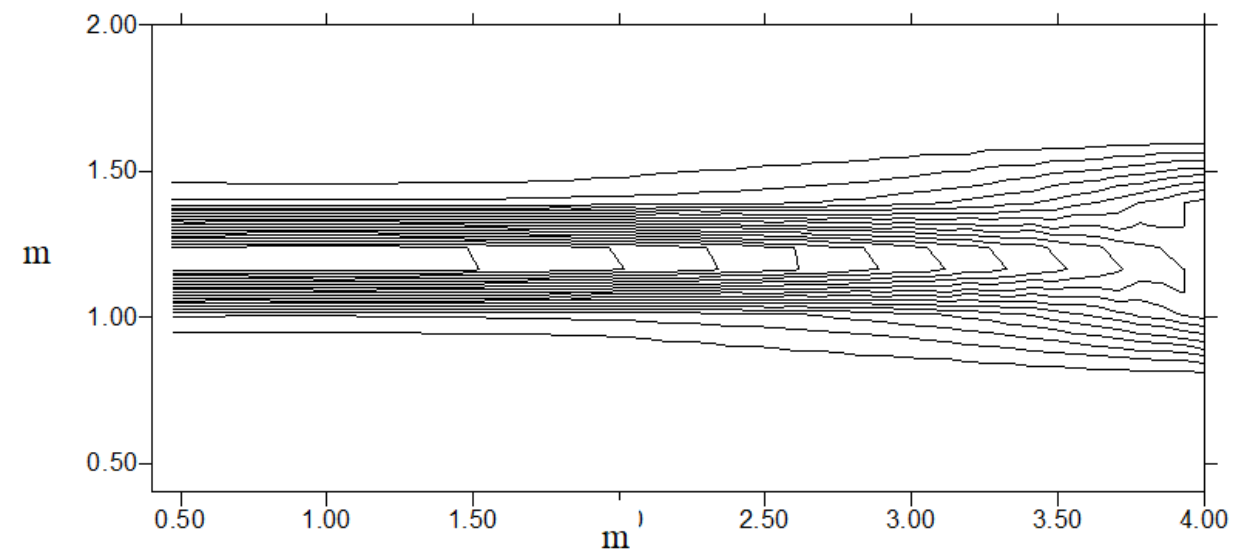

Fig.4. Separate stream flowing into the oncoming water stream. 
The lighter ozone-air mixture floats up in the surrounding water in the field of gravity. The diameter of the jet decreases, because of its acceleration. Outside the jet water flow is directed toward the bottom. Its speed is $0.02 \mathrm{~m} / \mathrm{s}$. The rate of the rising water-air mixture is directed to the surface and reaches $0.08 \mathrm{~m} / \mathrm{s}$. This speed is added to the speed of bubbles and reduces their time in water. For this reason, the fraction of insoluble ozone in the stream increases. Figure 5 shows the results of calculations of the fraction of insoluble ozone in the cross section of the water-air stream. In the center of the jet near the surface of the water, this fraction is 0.1 and is maximum for a given level.

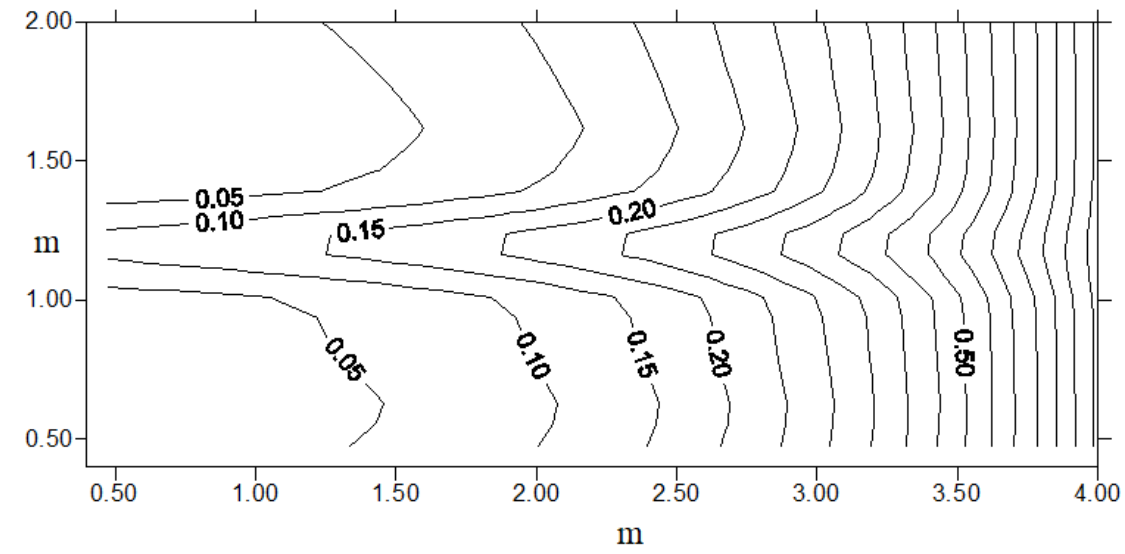

Fig.5. Contours of ozone concentration.

Figure 6 qualitatively shows the concentration of ozone on the surface of the tank for the pool section containing 25 dispersants. The figure shows that at the locations of the water-air mixture rising to the surface of the water, the fraction of insoluble ozone exceeds $10 \%$. At the same time, between the jets this fraction is close to zero. Obviously, a decrease in the efficiency of ozone dissolution in jets is connected with an increase in the rising bubble rate, which leads to a reduction of the time of ozonation.

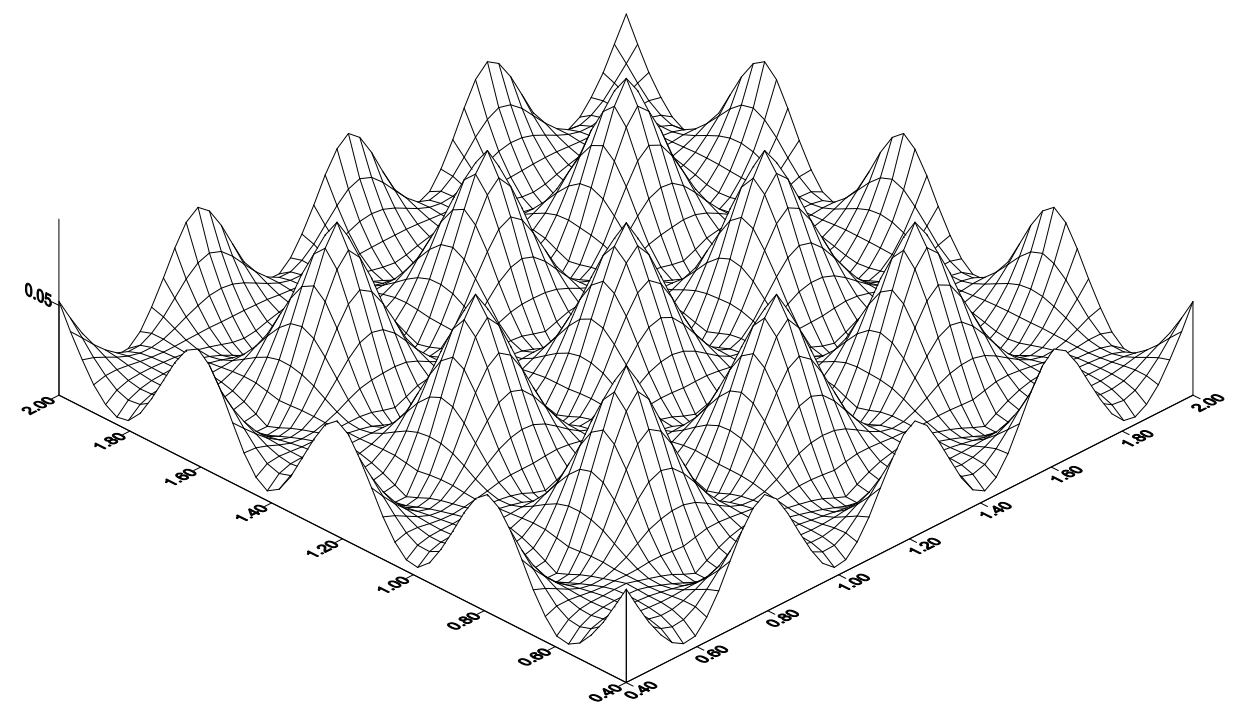

Fig.6. Isosurfaces of ozone concentration upon reaching the surface of the water in the contact tank.

Contact tanks with discretely located dispersants proved to be insufficiently effective. To compare the results we made the calculations, in which the sources of water and the ozone-air mixture were located continuously throughout the bottom of the pool. Figure 7 demonstrates the dependence of the levels of the relative fraction of insoluble ozone on the distance to the water surface for the case of continuously located sources. 


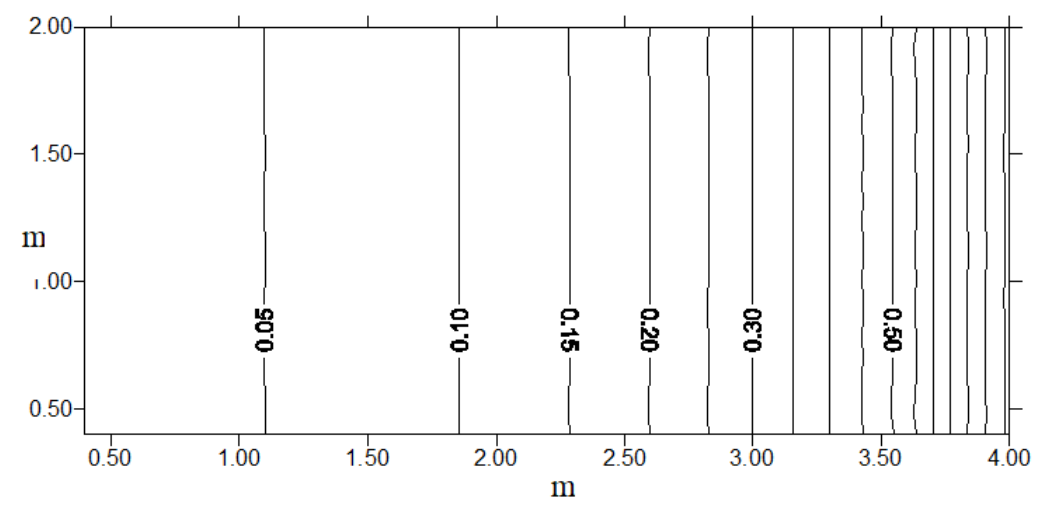

Fig.7. Degree of ozonation in the contact tank with uniform input ozone-air mixture.

The fraction of insoluble ozone on the surface is $2 \%$, which indicates a noticeable (compared to 10\%) increase in efficiency compared to the discrete location of sources.

\section{Conclusion}

The mathematical model used in the calculations, unlike the model of interpenetrating continuums for a two-phase medium, does not contain small parameters for derivatives and is much simpler from the point of view of numerical solution. By virtue of the consideration of the medium compressibility and the dependence of the density on the concentration of bubbles, this model automatically takes into account the processes causing free convection in the gravity field in the presence of the heterogeneous concentration of bubbles. This kind of convection, when the supply of gas mixture is not uniform in space, significantly affects the duration of stay of the bubbles in the reactor and, consequently, the completeness of the reactions that occur. An additional advantage of the proposed mathematical model is its analogy with compressible gas models. Going forward this analogy makes it possible to use well-developed numerical schemes for solving equations of gas dynamics. Parametric numerical studies, conducted according to the proposed model, have proved the efficiency of this approach. The acceleration of the floating of a gas jet due to its involvement in the motion of the carrier medium is confirmed. To determine the parameters of the ozone dissolution process during ozonation of water in contact tanks the preliminary numerical investigations have been carried out. The effectiveness of the location of the sources of ozone-air mixture at the bottom of the tanks has been evaluated.

\section{Acknowledgements}

The work was partially supported by the Ministry of Science and Higher Education of the Russian Federation (State Task No. 9.9625.2017/8.9).

\section{REFERENCES}

1 Kozhinov V.F., Kozhinov I.V. Water ozonation. M.: Stroiizdat, 1974, 496 p. [in Russian]

2 Orlov V.A. Water ozonation. - M.: Stroiizdat, 1984, 88 p. [in Russian]

3 Dankverts P.V. Gas-liquid reactions. M.: Himia, 1973. [in Russian]

4 Vasenin I.M., Dyachenko N.N., Elkin K.E., Narimanov R.K. Mathematical Modeling of Two-Phase Convective Flows with Fine Particles. Journal of Applied Mechanics and Technical Physics, 2004, No 6, pp. 788-793.

5 Vasenin I M, Narimanov R.K., Shrager L.A., Perchatkina E.V. Modeling of a two-phase flow of liquid with small-size gas bubbles. Eurasian Physical Technical Journal. 2019, Vol.16, No. 1(31), 2019, pp. 129-137.

6 Parkin B.R., Gilmor, F.R., Broud G.L. Shock waves in water with air bubbles. In the coll. Underwater and underground explosions. Moscow, Mir, 1974, pp. 152-258.[ in Russian]

7 Epstein, P. S. and Plesset, M. S. On the Stability of Gas Bubbles in Liquid-Gas Solutions. Journal of Chemical Physics. 1950, 18 (11). pp. 1505-1509. 